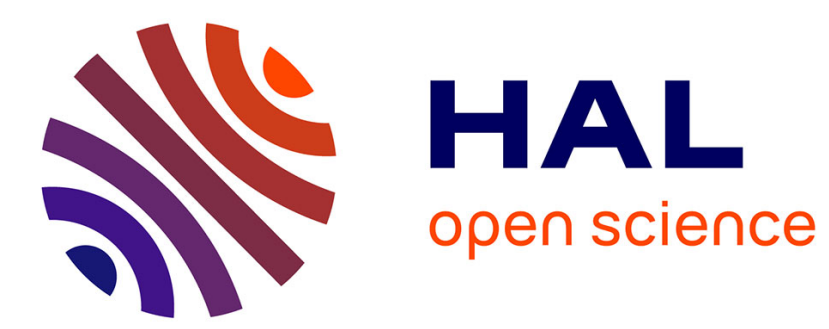

\title{
Dynamics of a ball on a vibrating plate
}

Pierre Devillard

\section{To cite this version:}

Pierre Devillard. Dynamics of a ball on a vibrating plate. Journal de Physique I, 1994, 4 (7), pp.10031011. 10.1051/jp1:1994180 . jpa-00246960

\section{HAL Id: jpa-00246960 https://hal.science/jpa-00246960}

Submitted on 1 Jan 1994

HAL is a multi-disciplinary open access archive for the deposit and dissemination of scientific research documents, whether they are published or not. The documents may come from teaching and research institutions in France or abroad, or from public or private research centers.
L'archive ouverte pluridisciplinaire HAL, est destinée au dépôt et à la diffusion de documents scientifiques de niveau recherche, publiés ou non, émanant des établissements d'enseignement et de recherche français ou étrangers, des laboratoires publics ou privés. 


\title{
Dynamics of a ball on a vibrating plate
}

\author{
P. Devillard
}

Centre de Physıque Théorıque de Marseılle, CNRS Luminy, Case y07, CPT, 13288 Marseılle Cedex 9, France

(Received 3 February 1994, received in final form 18 March 1994, accepted 23 March 1994)

\begin{abstract}
We study both analytically and numerically the problem of a partially melastic ball on a platform vibrating with frequency $\omega$. Two parameters control the dynamics, the restitution coefficient $\eta$, and the reduced acceleration $\Gamma=\gamma / g$, where $\gamma$ is the maximum acceleration of the motion of the plate and $g$ the acceleration of gravity. When $\eta$ exceeds some value, simple stable fixed points no longer exist, but it is shown that generic trajectories are periodic with period $T_{\mathrm{B}}$ which behaves as $T_{\mathrm{B}} \sim(1-\eta)^{-5}$ for $\eta$ close to 1
\end{abstract}

\section{Introduction.}

In this paper, we revisit the problem of one ball on a platform vibrating with period $T=2 \pi / \omega$ and maxımum amplitude $a[1-6]$, which was recently studied in reference [6]. In the following, we show that all the periodic motions, in some parameter regions, include the rest state, the state where the ball moves with the plate. The penod between a rest state and the next rest state, scales as $(1-\eta)^{-5}, \eta$ is the restitution coefficient.

\section{Description of the model.}

Instead of taking the movement of the platform to be sinusoidal, as in reference [6], we take it piecewise parabolic, with period $T$. Namely, the height $s(t)$ of the platform in the laboratory frame is, on each half period, an arch of a parabola, whose concavity is alternatively up and down (except at the very beginning, for $t \leqslant \frac{T}{2}$ ), as depicted in figure 1 . We have :

$$
\begin{array}{lll}
s(t)=\frac{\gamma}{2}\left(t-\frac{T}{2}\right)(T-t) & \text { for } & \frac{T}{2} \leqslant t \leqslant T, \\
s(t)=\frac{\gamma}{2}(T-t)\left(\frac{3 T}{2}-t\right) & \text { for } & T \leqslant t \leqslant \frac{3 T}{2}, \\
s(t+n T)=s(t) & \text { for } & t \geqslant \frac{T}{2}, n \in \mathbb{N} .
\end{array}
$$




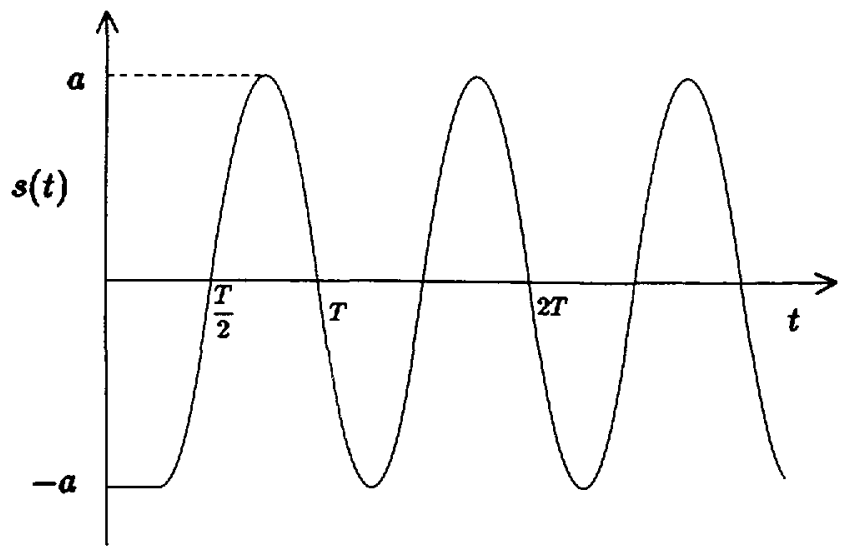

Fig. 1 - Position $s(t)$ of the vibratıng platform as a function of time $t$. For $t \geqslant \frac{T}{4}, s(t)$ is made of arches of parabola with second derivative $\frac{\mathrm{d}^{2} s}{\mathrm{~d} t^{2}}$ equal to $\gamma$ or $-\gamma$.

$\gamma$ is an acceleration which relates to the amplitude $a$ of the motion by

$$
a=\frac{\gamma T^{2}}{32}
$$

and the reduced acceleration $\Gamma$ will be defined by

$$
\Gamma=\gamma / g,
$$

where $g$ is the acceleration of gravity.

From now on, positions and velocities of the ball will not be counted in the laboratory frame, but in the reference frame of the moving platform, so that the position of the ball is also its height with respect to the platform and is always positive. In the reference frame of the platform, the force felt by a ball of mass one, when not in contact with the platform, is $-\frac{\mathrm{d}^{2} s}{\mathrm{~d} t^{2}}-g$. It takes alternatively the values $-\gamma-g$ and $\gamma-g$. We assume that we have a perfectly hard ball, and that $\Gamma$ is larger than 1 . We denote by $W^{+}$and $W^{-}$the relative velocities of the ball with respect to the platform respectively immediately before and after a bounce with the platform. The coefficient of restitution $\eta$ is defined by

$$
W^{+}=-\eta W^{-}
$$

\section{Description of the dynamics.}

We shall use two alternative ways of describing the dynamics. One is the standard way in the literature. The time $\tau$, where the $l$-th bounce occurs and $W_{l}^{+}$, the relative velocity of the ball just after the bounce, are recorded. A two dimensional map $\left(W_{l+1}^{+}, \tau_{l+1}\right)=f\left(W_{l}^{+}, \tau_{l}\right)$ is obtained, where $f$ is a function. This, however, is not convenient when the ball performs infinitely many hops and comes to rest on the platform. In this case, as in reference [6], we say that the ball lands in an absorbing region. It is relaunched later, when the force felt by the ball 
becomes positive, namely, when the driving acceleration, which is equal to $-\frac{\mathrm{d}^{2} s}{\mathrm{~d} t^{2}}$, overcomes gravity.

An alternatıve way is to simply record the height and velocity of the ball at each time the acceleration changes sign (at each half period). The acceleration felt by the ball will be alternatively $-\gamma-g$ and $\gamma-g$. In this descrption, $g$ will be set to one, the length of the halfperiod will be set to 1 , and accelerations will be counted in units of $\gamma+g$. The acceleration seen by the ball will thus alternatively take the values -1 and

$$
A=\frac{\Gamma-1}{\Gamma+1}
$$

Time is denoted by $t^{\prime}$ in this description. For any integer $l$, with $l \leqslant t^{\prime} \leqslant l+1$, the acceleration felt by the ball will be $A$. For $l+1 \leqslant t^{\prime} \leqslant l+2$, it will be -1 For every time $t^{\prime}$ equal to an even integer $2 n$, the positions and velocities at this time will be denoted by $x_{n}$ and $v_{n}$ respectively. It will turn out to be convenient to use the variables

and

$$
\begin{gathered}
Y_{n}=v_{n}, \\
X_{n}=\sqrt{v_{n}^{2}+2 x_{n}} .
\end{gathered}
$$

We also introduce $x_{n}^{\prime}$ and $v_{n}^{\prime}$, the positions and velocities of the ball at times $t^{\prime}=$ $2 n+1$ (i.e. every time the acceleration switches from positive to negative). $X_{n}^{\prime}$ and $Y_{n}^{\prime}$ are defined in analogy to $X_{n}$ and $Y_{n}$. The dynamics is now described by a map :

$$
\left(X_{n}, Y_{n}\right) \stackrel{h}{\rightarrow}\left(X_{n}^{\prime}, Y_{n}^{\prime}\right) \stackrel{h}{\rightarrow} \cdot\left(X_{n+1}, Y_{n+1}\right)
$$

The motion of the representative points $\left(X_{n}, Y_{n}\right)$ and $\left(X_{n}^{\prime}, Y_{n}^{\prime}\right)$ in phase space is restricted to the cones $\left(X_{n} \geqslant 0,\left|Y_{n}\right| \leqslant X_{n}\right)$ and $\left(X_{n}^{\prime} \geqslant 0,\left|Y_{n}^{\prime}\right| \leqslant X_{n}^{\prime}\right)$ respectively.

The precise form of the mapping $b$ and $h$ is a little complicated and not very informative, it is given in the appendix. One property of $h$ however is important. For any $\left(X_{n}^{\prime}, Y_{n}^{\prime}\right)$ such that

with

$$
\begin{gathered}
Y_{n}^{\prime}<1-r X_{n}^{\prime} \\
1=\frac{1+\eta}{1-\eta}, \\
X_{n+1}=Y_{n+1}=0 .
\end{gathered}
$$

That is to say, $h$ is identically zero. This corresponds to the absorbing region, the ball comes to rest on the platform.

\section{Scalıng of the period of the motion for large restitution coefficient.}

In this section, the period of the periodic motion for large restıtution coefficient is estımated. Starting from any initial condition, the ball always goes to the rest state, moving together with the plate. The regıme before the ball has reached the rest state for the first tıme will be called the transient regime. After it has reached the rest state for the first time, its movement will then be periodic. This is called the permanent regime. The number of iterations between two rest states, the period of the motion in this parameter region, is calculated as a function of both the restitution coefficient and the size of the permanent regime. The size of the permanent regime in turn, is also related to the value of the restitution coefficient. 
Having set the notations, we now give results about the dynamics. One issue is whether the ball generically lands in an absorbing region. If this occurs, then the subsequent motion will be periodic. It is simpler to discuss first a situation where a ball does not generically land in an absorbing region. Let us consider the mapping $\left(W_{l+1}^{+}, \tau_{l+1}\right)=f\left(W_{l}^{+}, \tau_{l}\right)$, which relates tımes and velocities just after the $(l+1)$-th bounce of the ball will the platform to times and velocities just after the $l$-th bounce. There may exist fixed points of the map, 1.e.: $\left(W_{l+1}^{+}=W_{l}^{+}\right.$and $\left.\tau_{l+1}=\tau_{l}\right)$. A fixed point with $W_{l+1}^{+}=W_{l}^{+}=0$ corresponds to the ball landing in the absorbing region. We call this kind of fixed point a null velocity simple fixed point. A fixed point with $W_{l}^{+} \neq 0$ will be called a non-zero velocity simple fixed point. If such a non-zero velocity simple fixed point exists, with $W_{l}^{+}=W_{0} \neq 0$, for example, and if it is stable, then there will be a neighbourhood of this point for which the ball will be attracted to it. For large $l, W_{l}^{+}$tends to $W_{0}$ and the ball will never land in an absorbing region. It is possible to enumerate all non-zero velocity simple fixed points, for a given value of $\eta$ and $\Gamma$, but the analytic expressions are moderately complicated and not particularly illumınatıng. One result is that for

$$
(1+\eta)^{2}\left(1+\eta^{2}\right)^{-1}>2 \Gamma^{-1},
$$

no stable non-zero velocity simple fixed points exist. Although the absence of such stable fixed points with $W_{l}^{+} \neq 0$ does not mean that a generic trajectory lands in an absorbing region, other stable attractors, fixed cycles, strange attractors, etc... are not observed in the numerical simulations for $\eta$ larger than 0.6 and $\Gamma$ larger than 2 . Thus, we shall assume that the only stable attractor, in this parameter region, is the rest state.

We now calculate the period of the motion (from one rest state to another), as a function of the restitution coefficient and of the size of the permanent regime. Later, we shall see that the permanent regime dynamics is confined to a regıon where $W^{+}<W_{\text {sup }}$, where $W_{\text {sup }}$ depends on $\eta$, if the dynamics is described by mapping $f$. If the description of the dynamics is done using mappings $b$ and $h$ (Eq. (6)), the permanent regime dynamics is confined to a region where $\sqrt{X^{\prime 2}+Y^{\prime 2}}<R_{0}$, where $R_{0}$ is a number which depends on $\eta$.

From equations (7a) to (7c), we see that a ball lands in the absorbing region if, and only if, it has not been absorbed before and $Y_{n}^{\prime} \leqslant 1-r X_{n}^{\prime}$. The region $Y_{n}^{\prime} \leqslant 1-r X_{n}^{\prime}$, and $\left|Y_{n}^{\prime}\right|<$ $X_{n}^{\prime}$ defines a triangle in $\left(X^{\prime}, Y^{\prime}\right)$ plane. In principle, it suffices to find the inverse iterates of this triangle $v i a$ the applications $b$ and $h$. We try to evaluate the area in $\left(X^{\prime}, Y^{\prime}\right)$ plane made by the points which are going to land in the absorbing region after $n$ periods of vibration of the platform, and not before. More precisely, if one starts with a point of coordinates $\left(X_{0}, Y_{0}\right)$, it transforms under $b$ into a point of coordinates $\left(X_{0}^{\prime}, Y_{0}^{\prime}\right)$ and lands in the absorbing region if $Y_{0}^{\prime} \leqslant 1-r X_{0}^{\prime}$. We say that the 0 th order absorbing zone, later denoted by $t_{0}$ is made by the rectangle triangle delimited by the straight lines: $Y_{0}^{\prime}=1-r X_{0}^{\prime}$, $Y_{0}^{\prime}=-X_{0}^{\prime}$ and $Y_{0}^{\prime}=X_{0}^{\prime}$. The $n$-th order absorption zone $t_{n}$, is defined in an analogous way as the points $X_{n}^{\prime}, Y_{n}^{\prime}$ which land in the absorbing region after $n$ steps, but have not landed there before ; 1 e., $Y_{n}^{\prime} \leqslant 1-r X_{n}^{\prime}$ and $Y_{s}^{\prime}>1-r X^{\prime}$, for any $s, 0 \leqslant s<n$.

The area of $\mathcal{A}_{0}$ is simply $(1-\eta)^{2} /(4 \eta)$. Since $b$ and $h$ are defined in a precewise fashion. $\mathcal{A}_{n}$ will be made in general of many patches. The Jacobian of $b^{-1}$, taken at the value $\left(X_{n}^{\prime}, Y_{n}^{\prime}\right)$, with $\left(X_{n}, Y_{n}\right)=b^{-1}\left(X_{n}^{\prime}, Y_{n}^{\prime}\right)$, will be denoted by $\mathfrak{J}\left(b^{-1}\right)\left(X_{n}^{\prime}, Y_{n}^{\prime}\right)$. It takes the value

$$
J\left(b^{-1}\right)\left(X_{n}^{\prime}, Y_{n}^{\prime}\right)=X_{n}^{\prime} / X_{n}, \quad \text { if } \quad Y_{n}^{\prime} \leqslant k\left(X_{n}^{\prime}\right),
$$

where

$$
k\left(X_{n}^{\prime}\right)=\sqrt{\frac{A}{A+1}} X_{n}^{\prime}, \quad \text { if } \quad X_{n}^{\prime}<\sqrt{A(A+1)}
$$


and

$$
\begin{gathered}
k\left(X_{n}^{\prime}\right)=\sqrt{X_{n}^{\prime 2}+A+1}-1, \quad \text { if } \quad X_{n}^{\prime} \geqslant \sqrt{A(A+1)} . \\
J\left(b^{-1}\right)\left(X_{n}^{\prime}, Y_{n}^{\prime}\right)=\eta^{-2} X_{n}^{\prime} / X_{n}, \quad \text { if } \quad Y_{n}^{\prime}>k\left(X_{n}^{\prime}\right) .
\end{gathered}
$$

We have also $J\left(h^{-1}\right)=\eta^{-m}$, where $m$ is defined in equation (A.2f) of the appendix. As $\eta$ tends to 1 , the area of $\mathfrak{t}_{0}$ shrinks as $(1-\eta)^{2}$ and the avallable phase space grows like $R_{0}^{2}$, where $R_{0}$ is the size of the region where the permanent regime dynamics takes place.

We only give here a simplified backbone of the argument. The $t_{n}$ zones gradually cover all the available phase space as $n$ increase. By definttion, the $t_{n}$ zones are disjoint. Otherwise, if two $t_{n}$ and $\mathfrak{A}_{m}$ with $n \neq m$ overlapped, the ball would land for the first time in the absorbing region after $n$ steps and also after $m$ steps, which is impossible.

If $t_{n}$ is mainly built up by points located at a distance of order $z$ from the origin in $\left(X^{\prime}, Y^{\prime}\right)$ plane, the presence of the factor $X_{n}^{\prime} / X_{n}$ in the Jacobian (Eq. (9a)) implies that the area of the $\mathfrak{t}_{n}$ zone shrinks by a factor $\mathscr{F}_{n}=\frac{X_{n}^{\prime} X_{n-1}^{\prime} X_{n-2}^{\prime} \ldots X_{0}^{\prime}}{X_{n} X_{n-1} X_{n-2} . X_{0}}$, with $X_{n}^{\prime} \sim z$ and $X_{n} \sim z$. In the regions where the main part of the dynamics takes place, we have $X_{n}^{\prime}=X_{n-1}$. This can be seen from equations (A.2d) to (A.2g) of the appendix, giving the precise from of the mapping $h$; we have almost all the time $m=0$ in equation (A.2f). The shrink factor $\mathcal{F}_{n}$ will thus be of order $\frac{X_{0}^{\prime}}{X_{n}}$, which behaves as $z^{-1}$.

The area of the $t_{n}$ zone shrinks thus as $z^{-1}$ Since every point belongs to only one $t_{n}$ zone, the number $l$ of iterations necessary to cover all the accessible phase space, where the permanent regime takes place, whose area is of order $R_{0}^{2}$, behaves as

$$
I \sim \int_{0}^{R_{0}} \frac{2 \pi z}{(1-\eta)^{2} z^{-1}} \mathrm{~d} z \sim(1-\eta)^{-2} R_{0}^{3} .
$$

We give a few words of explanation about this formula. One starts with $\mathfrak{t}_{0}$, whose area is already of order $(1-\eta)^{2}$. The elementary portion of phase space, in $\left(X^{\prime}, Y^{\prime}\right)$ plane limited by the two diagonals $Y^{\prime}=X^{\prime}, Y^{\prime}=-X^{\prime}$ and by the two circles centered at the origin and of radii $z$ and $z+\mathrm{d} z$, will have to be covered by $\mathfrak{t}_{n}$ zones. The area of one particular $t_{n}$ zone is of order $(1-\eta)^{2} z^{-1}$ and the area to be covered is $2 \pi z \mathrm{~d} z$. It thus takes $\frac{2 \pi z \mathrm{~d} z}{(1-\eta)^{2} z^{-1}}$ iterations to cover this elementary portion of phase space. Since all the accessible phase space must eventually be covered, equation (10) follows. We are thus left with the task of estimating $R_{0}$.

The typical size of the region of phase space where the permanent regime takes place has been already studied for a model slightly different from ours, in reference [6]. Applying their method to our case, the permanent regime dynamics will be confined to the region $W^{+}<W_{\text {sup }}$, with

$$
W_{\text {sup }}=\frac{\eta \gamma T}{4(1-\eta)(1+\eta)}\left(\eta+3+\sqrt{(1+3 \eta)^{2}+\frac{4}{\Gamma}\left(1-\eta^{2}\right)}\right) .
$$

We have the limiting behaviour

$$
W_{\text {sup }} \sim(1-\eta)^{-1} \text { for } \eta \rightarrow 1 .
$$

This means that an upper bound on the velocities is of order $(1-\eta)^{-1}$ Integration of the equations of motion between successive bounces with the platform show that time between 
two consecutive bounces will be at most of order $(1-\eta)^{-1}$, and that maximum heights reached by the ball will be at most of order $(1-\eta)^{-2}$ The maximum values of the velocity of the ball, taken at times where the acceleration changes sign will also be at most of order $(1-\eta)^{-1}$ These are precisely the values of $v_{n}$ and $v_{m}^{\prime}$, used in the description of the dynamics by mappings $b$ and $h$. Thus an upper bound on $v_{n}$ or $v_{n}^{\prime}$ is of order $(1-\eta)^{-1}$. An upper bound on $x_{n}$, or $x_{n}^{\prime}$ s of order $(1-\eta)^{-2}$ By definition of $X_{n}^{\prime}$ and $Y_{n}^{\prime}$ (Eqs. (5a) and (5b)), the maximum values of $X_{n}^{\prime}$ and $Y_{n}^{\prime}$ behave at most as $(1-\eta)^{-1}$ Thus, the dynamics 1 confined to a region of $\left(X^{\prime}, Y^{\prime}\right)$ plane where $X^{\prime}<X_{\text {sup }}^{\prime}$, with $X_{\text {sup }}^{\prime} \sim(1-\eta)^{-1}$.

Since this is only an upper bound, we checked numerically that the typical size $R_{0}$ of the domain where the dynamics takes place in the permanent regime scales as $(1-\eta)^{-1}$ for $\eta \rightarrow 1$ We take a ball at the origin $\left(X^{\prime}, Y^{\prime}\right)=(0,0)$ and integrate the dynamics. We study $R_{0}$, the maximum value of $\sqrt{X^{\prime 2}+Y^{\prime 2}}$ reached by the ball. Since the values of $R_{0}$ strongly depend upon $A$, averages have been taken over typically $10^{2}$ trajectories, with different values of $A$ in intervals $\left[A_{\min }, A_{\max }\right]$. We did not have to average on $\eta$. Figure 2 shows a $\log -\log$ plot of $R_{0}$ as a function of $(1-\eta)^{-1}(1-A)^{-1 / 2}$, for $\eta$ between 0.5 and 0.9 and ( $1-A$ ) between $10^{-4}$ and $10^{-1} R_{0}$ behaves thus as $(1-\eta)^{-1}$ for $\eta \rightarrow 1$.

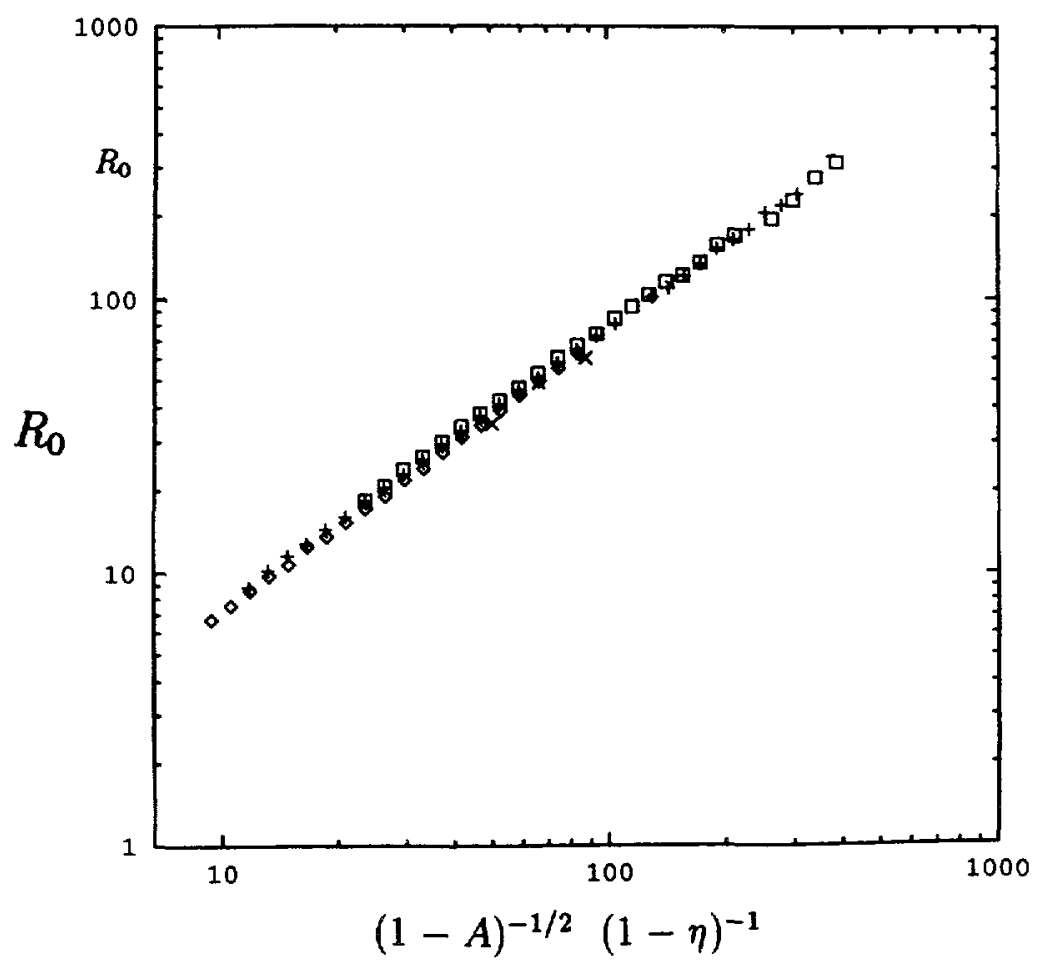

Fig. 2. - Log-log plot of $R_{0}$ the typical size of the phase space region visited in the permanent regime (see text) versus $(1-A){ }^{1 / 7}(1-\eta)^{-1}$, for values of $\eta=0.5(\ominus), \eta=0.6(+), \eta=0.8(\square)$, and $\eta=0.9(\times)$. Averages have been taken over typically $10^{2}$ values of $A$. For example, for the point centered at $(1-A)=7.5 \times 10^{-4}$, and $\eta=0.6$, averages have been taken over 100 values of $(1-A)$ ranging from $(1-A)=5 \times 10^{-4}$ to $(1-A)=11 \times 10^{-3}$ A least square fit to the data gives a slope 101 . The uncertainty on the slope is about 0.1 
From equation (10), the number $l$ of iterations necessary to cover all the accessible phase space behaves as

$$
l \sim(1-\eta)^{-5}
$$

We performed numerical simulations, starting from a ball at rest. We determined the first time $T_{\mathrm{B}}$ for which the ball comes to rest on the platform. Because $T_{\mathrm{B}}$ very strongly depends on $\Gamma$, results have been averaged over typically $10^{3}$ values of $\Gamma$ in intervals $[\Gamma-\delta \Gamma$, $\Gamma+\delta \Gamma$ ], with $\delta \Gamma<\Gamma$. A Log-log of $T_{\mathrm{B}}$ versus $(1-\eta)^{-5} \Gamma^{-1}$, for two values of $\Gamma$ is shown in figure 3. Least square fits to the data give slopes $1.002 \pm 0.02$ for $\Gamma$ around 7.8 and $0.985 \pm 0.03$ for $\Gamma$ around 15.8. Moreover, data seem to collapse on a single curve.

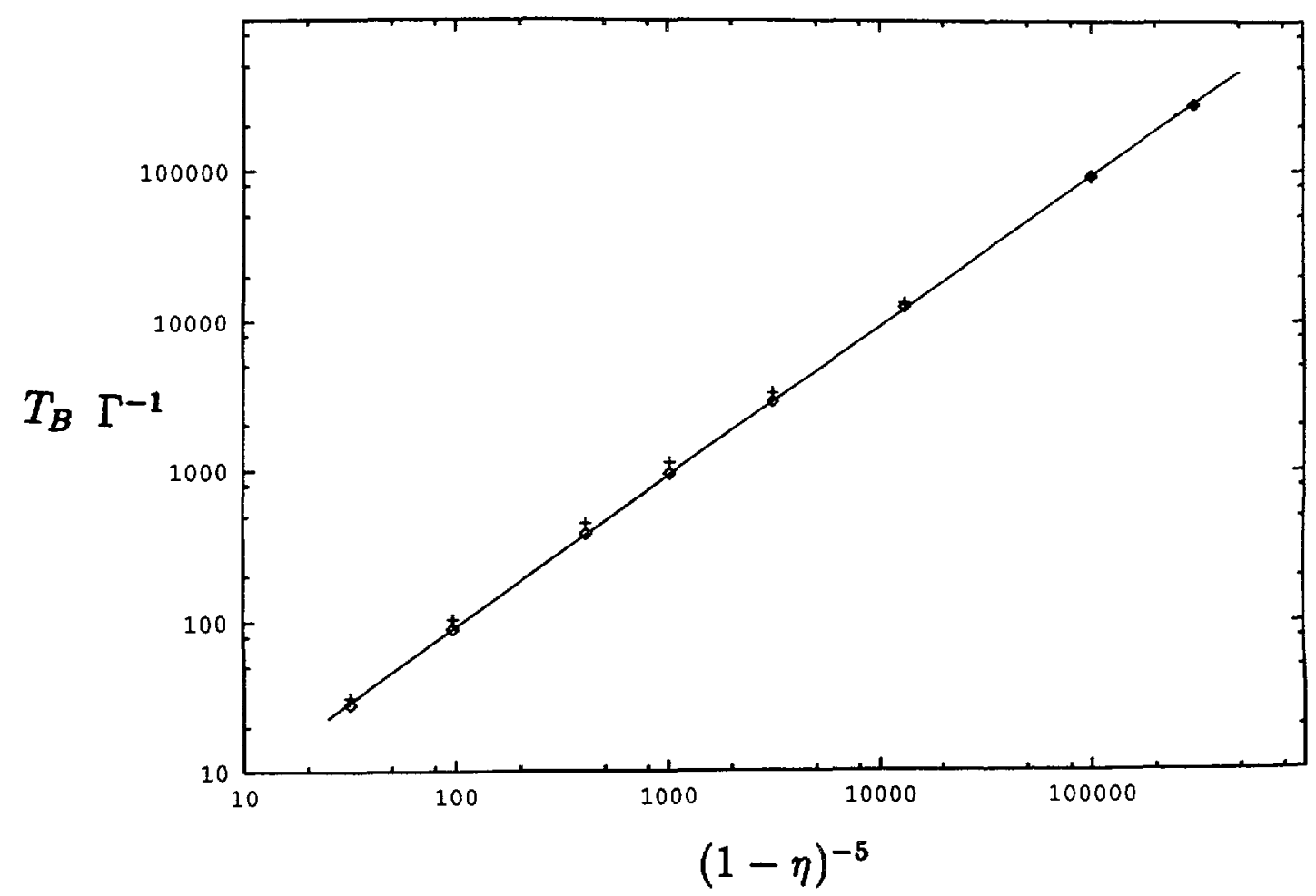

Fig. 3. - $\log$ - $\log$ plot of the ratio $T_{\mathrm{B}} \Gamma^{-1}$, where $T_{\mathrm{B}}$ is the period of the movement of the ball and $\Gamma$ the reduced acceleration versus $(1-\eta)^{-5}$ for two values of $\Gamma,(\odot) 7.4 \leqslant \Gamma \leqslant 8.2$ and $(+) 15.4 \leqslant$ $\Gamma \leqslant 16.2$. Each point corresponds to averages over typically $10^{3}$ values of $\Gamma$ and one definite value of $\eta$. The straight line is a least square fit to the data for $7.4 \leqslant \Gamma \leqslant 8.2$ and has a slope 1002 .

\section{Conclusion.}

We have introduced a simplified version of the partially inelastic bouncing ball on a vibrating platform which enables the calculation of fixed points. For an acceleration larger than $2 g$, beyond some restitution coefficient $\eta=0.6$, all trajectories seem to end up to be periodic. However, the period can be shown to diverge as $(1-\eta)^{-5}$ for $\eta \rightarrow 1$, and as $\Gamma$ for $\Gamma \rightarrow \infty$. 


\section{Acknowledgements.}

We thank J. M. Luck for very useful discussions and the region P.A.C.A. (Provence, Alpes, Côte d'Azur) for generous allocations of computer time on the Cray YMP 2E of the I.M.T. (Institut méditerranéen de technologie).

\section{Appendix.}

We give below the exact expressions for the mappings $b$ and $h$ which appear in (6).

The mapping $b$ is defined by:

$$
\begin{aligned}
& X_{n}^{\prime}=\sqrt{X_{n}^{2}+2(A+1) Y_{n}+A(A+1)}, \\
& Y_{n}^{\prime}=Y_{n}+A, \\
& \text { if } X_{n}<\sqrt{A(A+1)} \text { and } Y_{n}>-\sqrt{\frac{A}{A+1} X_{n},} \\
& \text { or if } \quad X_{n}>\sqrt{A(A+1)} \text { and } Y_{n}>1-\sqrt{A+1+X_{n}^{2}},
\end{aligned}
$$

and, otherwise, by

$$
\begin{aligned}
& X_{n}^{\prime}=\sqrt{\frac{A+1}{A} Y_{n}^{\prime 2}-\frac{\eta^{2}}{A}\left[(A+1) Y_{n}^{2}-A X_{n}^{2}\right]} \\
& Y_{n}^{\prime}=Y_{n}+A+(1+\eta) \sqrt{(A+1) Y_{n}^{2}-A X_{n}^{2}}
\end{aligned}
$$

The mapping $h$ is defined, if $X_{n}^{\prime} \neq 0$ by :

$$
\begin{gathered}
X_{n+1}=Y_{n+1}=0, \\
\text { if } \quad Y_{n}^{\prime}<1-r X_{n}^{\prime},
\end{gathered}
$$

where

$$
r=\frac{1+\eta}{1-\eta}
$$

This corresponds to the absorbing region.

Otherwise, $h$ is defined by :

$$
\begin{aligned}
& X_{n+1}=\eta^{m} X_{n}^{\prime}, \\
& Y_{n+1}=Y_{n}^{\prime}-1+\left\{1+2 \frac{\eta-\eta^{m}}{1-\eta}+\eta^{m}\right\} X_{n}^{\prime},
\end{aligned}
$$

with

$$
m=\operatorname{Int}\left\{\operatorname{Ln}\left[\left(\frac{1+\eta}{2}\right)-\left(\frac{1-\eta}{2}\right) Q^{\prime}\right] / \operatorname{Ln} \eta\right\}
$$

and

$$
Q^{\prime}=\frac{1-Y_{n}^{\prime}}{X_{n}^{\prime}}
$$

«Int » denotes the integer part of a real number. 


\section{References}

[1] Fermı E., Phys. Rev. 75 (1949) 1169.

[2] Kowalik Z. J., Franaszek M. and Pieranski P., Phys. Rev. A 37 (1988) 4016.

[3] Everson R. M., Physica 19D (1986) 355.

[4] Celaschi S. and Zimmerman R. L., Phys. Lett. A 120 (1987) 447.

[5] Mehta A. and Luck J. M.. Phys. Rev. Lett. 65 (1990) 393-396.

[6] Luck J. M. and Mehta A., Phys. Rev. E 48 (1993) 3988. 